\title{
The Evolution of Chatbots in Tourism: A Systematic Literature Review
}

\author{
Davide Calvaresi ${ }^{(凶)}$, Ahmed Ibrahim, Jean-Paul Calbimonte, Roland Schegg, \\ Emmanuel Fragniere, and Michael Schumacher \\ Institute of Information Systems, University of Applied Sciences and Arts Western \\ Switzerland (HES-SO), Sierre, Switzerland \\ \{Davide.Calvaresi, Ahmed. Ibrahim, Jean-Paul.Calbimonte, Roland.Schegg, \\ Emmanuel.Fragniere, Michael. Schumacher\}@hevs.ch
}

\begin{abstract}
In the last decade, Information and Communication Technologies have revolutionized the tourism and hospitality sector. One of the latest innovations shaping new dynamics and fostering a remarkable behavioral change in the interaction between the service provider and the tourist is the employment of increasingly sophisticated chatbots. This work analyzes the most recent systems presented in the literature (since 2016) investigated via 12 research questions. The often appreciated quick evolution of such solutions is the primary outcome. However, such technological and financial fast-pace requires continuous investments, upskilling, and system innovation to tackle the eTourism challenges, which are shifting towards new dimensions.
\end{abstract}

Keywords: Chatbots $\cdot$ Tourism $\cdot$ Virtual assistants $\cdot$ Virtual agents · Hospitality $\cdot$ eTourism

\section{Introduction}

The fast-paced evolution of Information and Communication Technologies (ICTs) has radically transformed the dynamics and business models of the tourism and hospitality industry [32]. This leads to new levels/forms of competitiveness among service providers and transforms the customer experience through new services. Creating unique experiences and providing convenient services to customers leads to satisfaction and, eventually, customer loyalty to the service provider or brand (i.e., hotels) [4]. In particular, the most recent technological boost received by the tourism sector is represented by mobile applications [16]. Indeed, empowering tourists with mobile access to services such as hotel reservations, airline ticketing, and recommendations for local attractions generates fervent interest and considerable revenues $[8,37]$.

On the one hand, immediate access, automation, and ease of use have made these applications an irreplaceable part of many tourists' daily lives. On the other hand, beyond automation-related features, there is a need for personalization. To do so, several Artificial Intelligence (AI)-based solutions (e.g., chatbots) 
are getting space in the market [2]. A chatbot is a computer program able to entertain a natural language-based conversation with a human. The ancestor of modern chatbots dates back to the 60s when Joseph Weizenbaum developed ELIZA [38]. Its goal was to simulate a psychotherapist with a bounded knowledge and several workarounds to avoid dead-ends in the conversation. Although more than 50 years have passed since that revolutionary idea, chatbot technologies (CBTs) have only recently reached sufficient maturity to be widely deployed and used in diverse real-life scenarios. Today, chatbots are intended to be programs understanding one or more human languages by using Natural Language Processing (NLP) or AI Markup Languages leveraging on a knowledge-base consisting of a collection of dialogue management rules that use different techniques for processing the user's input [37].

In the last five years, early prototypes were mainly based on simple state machines, offering simple interactions simulating conversations with humans $[2,7]$. In the tourism sector, the first interactions delegated to a chatbot were used to support the search for tips and information (e.g., opening hours) of local restaurants [17] and customer-care basic support (i.e., 85\% of customer care in tourism are today handled by chatbots/AI-based systems [37]). Besides the main characteristic of CBT (i.e., anytime-anywhere availability) and the main objective (i.e., providing information) chatbots have also been used for data collection. In the era of data-driven AI, this capability is priceless, enabling the provision of tailored recommendations and dialogues, which were/are expected to boost the user experience. For example, in the hospitality sector, Mercure, the AccorHotels brand, has chosen Facebook Messenger to host its virtual assistant. Guests can discover anecdotes about the surrounding area and secret addresses thanks to geolocation. Booking.com's new service and support chatbot is now widely available to English-language bookings, handling $30 \%$ of those customer questions automatically in less than five minutes.

In the context of the tourism industry, to provide a reconciling view on the most advanced solutions presented in the literature, we aim at analyzing how far have the current solutions and research gone? and what is targeted or envisioned by the tourism sector and the related research? This study aims at fostering the understanding of what stands behind those interactive dialogues between chatbot technologies and customers, beyond the well-known buying tickets online or book hotels support in the tourism industry. To do so, we have conducted a Systematic Literature Review, following a welldefined methodology. The methodology identifies a series of research questions against which existing works are analyzed.

The remainder of the paper is organized as follows. Section 2 presents the review methodology employed in this study. Section 3 reports the results and evidence. Section 4 discusses the aggregated and generated knowledge produced by this study. Finally, Sect. 5 concludes the paper. 


\section{Review Methodology}

This paper adheres to the original procedure for literature review presented in [24] and further adopted and adapted by [5] and [6] (see Fig. 1). This methodology applied to conduct the Systematic Literature Review (SLR) is meant to be rigorous and reproducible (i.e., replicate the retrieval, selection, and analysis processes).

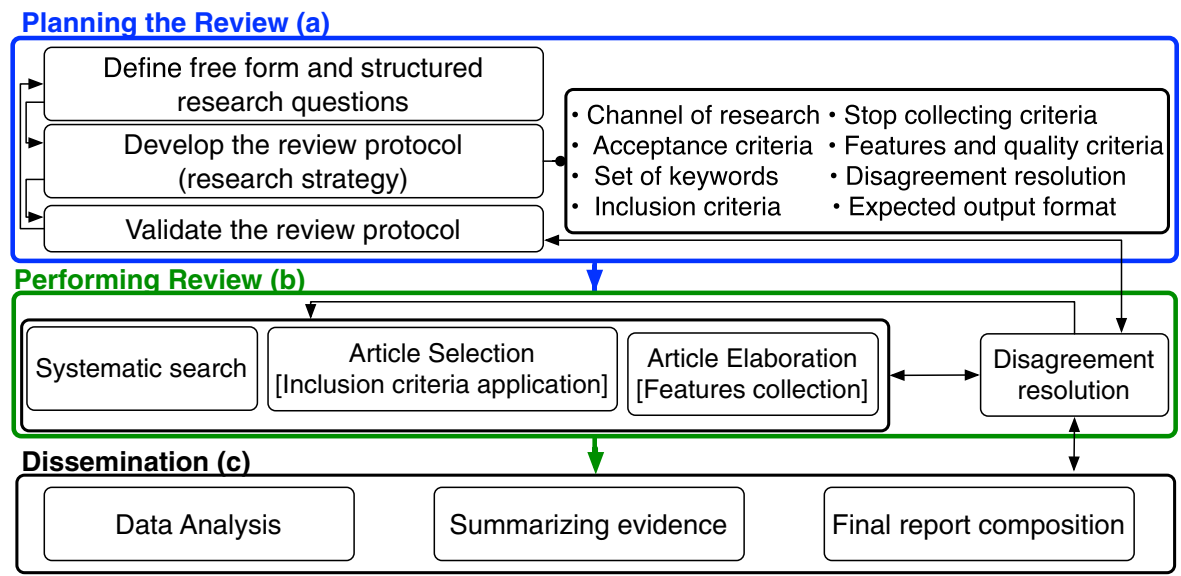

Fig. 1. Review methodology adapted from [5].

Following the Goal-Question-Metric (GQM) [25], the generic free-form question "How is the evolution of chatbots in Tourism characterized?" is broken down into the following structured research questions (SRQs):

- SRQ1: Demographics. How time- and geographic-wise are the research efforts distributed? i.e., when (year) and where (the geographical indication of the scientific institute).

- SRQ2: Abstraction. What is the abstraction level of the elaborated scientific contributions? e.g., at which level the contribution is: conceptual (C), prototype $(\mathrm{P})$, or tested $(\mathrm{T})$.

- SRQ2: Application scenarios. Which applications/areas of the tourism domain have employed CBT-solutions? (e.g., hospitality, travel agency, and transportation).

- SRQ3: Recipients. Who are the users of CBT-solutions?

- SQR4: Desiderata. Which are the requirements standing behind the employment of CBT?

- SQR5: Goals. Which are the objectives set for CBT-solutions?

- SQR6: Services realized. Which CBT functionalities have been realized?

- SQR\%: Services envisioned. Which CBT functionalities are desired and envisioned? 
- SQR8: Technology. Which underlying technologies have been employed to realize the CBTs?

- SQR 9: Benefits. Which advantages do CBTs provide? (from both user and provider sanding points).

- SQR10: Drawbacks. Which limitations have CBTs shown?

- SQR11: Open challenges. Which open challenges concern the next generation of CBTs?

To increase the accuracy of the semi-automatic research, some keywords have been contextualized (i.e., some contextual words have been associated with queried keywords). In particular, the queries have been realized by combining the two sets listed below:

- Contextual keywords: tourism + hospitality + traveling;

- Targeted keywords: chatbot + virtual assistant + online assistant + automated assistance + conversational agent.

The research of the articles has been conducted using the following sources: IEEExplore, Science Direct, ACM Library, and Google Scholar. Ninety-three relevant papers have been initially collected. Performing a coarse-grained and successively fine-grained examination, the primary studies to be elaborated have been reduced to 27 . Such filtering has been performed by briefly parsing the title, abstract, and the core contribution of the paper, which had to comply with the following criteria:

a) Recency (post-2016): The aim is to identify the current trends and understand recent works addressing CBT in tourism. Given the recent technological advancement and the tangible contribution of chatbots to the tourism industry, we set 2016 as the starting year of the collection.

b) Relevance: The paper must confer relevant information and contribution to the tourism sector (solely scholarly papers without a link to the tourism domain have been excluded).

c) Accessibility: To be included, the content of the article should be accessible via one of the portals mentioned above.

d) Singularity/Originality: Duplicate papers or papers having an extended follow-up version are not included. Only the complete version is included.

\section{Results Presentation}

\section{SRQ1 and SRQ2: Demographics and Abstraction}

The paper selection and elaboration have been conducted in late July 2020. That justifies the only 11 papers collected in that year. However, the projection suggests the exponential trend, manifesting a significant interest from the scientific community.

Figure 2(b) shows the papers distribution per country. The abstraction level of the elaborated studies is quite significant. Indeed, most of them propose practical and tested solutions (16 studies), only five studies present systems at a prototype level, and six solely conceptual contributions. 


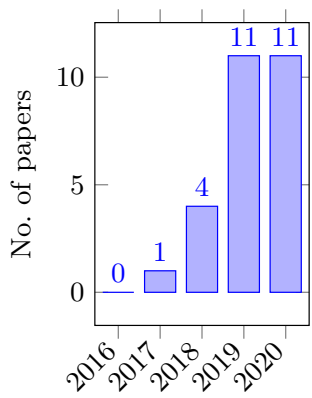

(a)

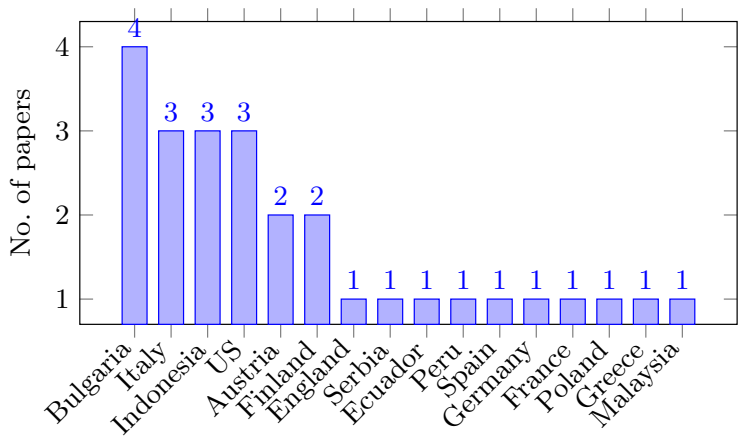

(b)

Fig. 2. (a) Number of papers per year. (b) Number of papers per country.

\section{SRQ3 and SRQ4: Application Scenario and Recipients}

The majority of the elaborated studies focus on hotel, airline, and travel agency sub-domains (20 studies). Four focus on promoting specific areas or cultural heritage sites (e.g., CBT for promoting the city of Manta in Ecuador [2] and Pompeii's archaeological park in Italy [26]). Finally, one study focuses on medical tourism (i.e., a chatbot identifying medicines available in the visited location compared with those, possibly unavailable, sold in the tourist's origin location).

All the elaborated studies aim to smoothly handle high volumes of customers [37], simplify the use of chatbots for end-users (i.e., investigating and identifying new ways to drive the user through a booking [32]), finding the right answers [33] and the right -tone-aware- approach [18]) and satisfying the functional requirements indicated by the service providers (i.e., enhance the system performance [3], automating and testing new functionalities [3], and improve the data collection (i.e., preferences and feedback $[18,37]$ ). Although in most cases, the chatbots aim at satisfying any kind of user interacting with them, some tourism offices targeted the Millennials as an unquestionable vector of information and technology itself, impending dedicated chatbot-campaigns over social media and a major messaging platform (i.e., Telegram) [1].

\section{SRQ5: Desiderata}

The requirements elicited from the primary studies have been classified into four categories: Financial (F), Technological (T), Socio-Technical (ST), and SocioManagement (SM). Implementing a competitive solution can require considerable financial investments [4] For example, the costs can vary between $\$ 30.000$ and $\$ 150.000$ [27]. Moreover, to gather the data needed for the user request, many CBTs make commercial use of services such as Avis, Uber, IBM Watson, and Google Dialogflow, and Google Maps, which also require financial expenses [2].

CBTs also require a remarkable technical knowledge. For example, modelling user and system dynamics [3], identifying and designing the right architecture [2], 
modelling and automating processes and testing [3,29], and modelling and implementing data collection, compliance, and organization $[2,17,35]$.

From a socio-technical point of view, the semantic interactions demand most of the effort. For example, enhance interactions when dealing with structured FAQs in more dynamic, explicative, and user-friendly manners [1,4]. Identify the conversations' tones are of paramount benefit for customer care. Indeed, $\mathrm{Hu}$ et al. [18] highlighted the significance and impact of using different tones in the context of social media customer care. Moreover, it can be mentioned defining, classifying, and representing the context (i.e., via context dimension tree) $[11,26]$. Furthermore, seamlessly transferring the conversation from the chatbot to a human operator agent is extremely needed if it is stalled [17]. Finally, other issues to mention are solving ambiguities, data and error handling [17], and monitoring and evaluating the chatbot effectiveness and efficiency [3].

The socio-management requirements concern the procedural and management qualifications, as well as the user-based analysis to be conducted for a more personalized experience and marketing response $[19,21,26]$. In particular, it can be mentioned maintenance and update of the service manuals and all concerned staff about the relevant changes of the system [21]. Furthermore, training staff to use the system at its best and addressing their concerns and resistance to change is inevitable. [21]. In turn, developing a marketing communications program to inform customers, suppliers, and other stakeholders on the changes [21] become priority requirements. Finally, data analysis is crucial to better understanding users' and the market's behavior [29].

\section{SRQ6: Goals}

To have a better understanding of the goals of these chatbots, we classified the papers in Industry-related (i.e., 9 studies mainly focusing on the current state of CBTs in the tourism industry), and purely Academic-related (i.e., 11 studies focusing on the technical aspects and the development of the chatbot itself).

Promoting a seamless and automated anytime-anywhere support for the tourists vising cultural and heritage sites is a common objective (i.e., city of Manta in Ecuador [2] and Pompeii's archaeological park in Italy [26]). Moreover, providing local information is also combined with the need for leveraging on social media (i.e., using the Messenger platform to provide continuous interactive tourism information about Yogyakarta [1]). Given that CBTs are evolving at a fast pace, to deliver incremental functionalities or adding new ones to enhance the user experience has often been set as a priority goal. Examples include creating a novel tone-aware chatbot that generates toned responses to user requests [18], automatizing testing the functionalities of CBTs [3], introducing a chatbot based on a context-aware system able to recommend contents and services to increase the promotion of cultural heritage [11], or the realization of a companion chatbot to help travelers decoding medical drug boxes sold in the host country, linking them with the corresponding trade name sold in the traveler's home country [35]. 


\section{SRQ7: Services Realized}

The services presented by the elaborated studies can be classified as purely technological and socio-technical functionalities. Concerning the technological ones, they focus mainly on the back-ends (i.e., functionalities inside the chatbot) that are not interacting nor visible to the end-user. For instance, we can cite mining and manipulating the acquired data [36] or automating the tests of chatbots through emulation using Java-based implementations that automatically parse plans and generate concrete test cases at run-time [3]. Moreover, the architecture in [2] aims at extracting the user's intent and expectations searching for text patterns in the user's messages, and using more advanced AI techniques applied to human conversation. Finally, other services to mention are hotel-related forecasting (i.e., tourist arrivals, demand, and hotel occupancy) and analyzing the impact of online reviews on hotel performance to offer the provider a better and clearer vision in the long run [19]. In general, chatbot interfaces are just composed of the chat showing the messages exchanged and the keyboard or the interaction menu prizing simplicity and efficiency. Nevertheless, a few applications opted for dedicating an important portion of the screen/window to the profile picture (i.e., a cartoonized icon of a flight assistant [23]).

On the one hand, the socio-technical functionalities (STF) address what can be related to the service management by performing basic tasks such as booking a room, answering FAQs $[4,12,33]$, understand and answer customer queries instantly [33], ordering meals or drinks [31], controlling the room temperature, lighting, taxi booking, and itinerary planning [10,31], and identifying a corresponding medical product from the user's home market [35]. On the other hand, they can solely communicate to the client messages pre-arrival, throughout their stay and post-checkout [4], generating toned responses to user requests based on their humor using the seq2seq model implemented with recurrent neural networks (RNN), such as the Long Short-Term Memory (LSTM) or the Gated Recurrent Units (GRU) model [18], providing necessary information to offer a better touristic experience [26], even adapting the user interface according to the visitor's backgrounds for better personalization [11].

Marketing and sales play a significant role in the STF of a CB as they can create personalized travel recommendations of touristic sites and attractions $[2,32]$, promote marketing campaign based on consumer involvement [1], suggest special dishes [31], greet guests at check-in at the hotel and remind them about the services available in the hotel [21]. Finally, analysis tools are strategic for a CB. Indeed, they allow the provider to extract the user's intent and expectations and to identify the user's preferences [2], to learn, based on previous choices made by the visitor, what information he/she can be further interested in [11], to forecast tourism arrivals, demand, and hotel occupancy, and to analyze the impact of online reviews on hotel performance [19].

\section{SRQ8: Services Envisioned}

The functionalities which have not been designed/implemented - yet conceivedhave been classified as socio-technical and technological. The envisioned sociotechnical functionalities concern personalization by tailoring guest's stay and 
experience and integrating voice command functions [4], enhancing the level of interaction with tourists during their visits and acting as a personal guide [2]. Other examples are smart hotel rooms' amenities and services customization directly via chatbots [4], using AI and ML for emotions-based mechanisms to develop proactive chatbots [4], training the chatbot to learn the styles characterizing different brands and behave accordingly [18], training the system with new heterogeneous sources of data and services, more complex environments and improvements based on the feedback received [11]. The envisioned technological functionalities focus on the automation side, leveraging on extensive testing to achieve more generalizable approaches [3] and studying the principles and pillars of CBT - enabling a deeper understanding of the technology can enhance future solutions for modern needs [17].

\section{SRQ9: Technology}

The spectrum of the technologies employed is quite broad. Many systems have stand-alone back-ends, entirely developed/commissioned by the service provider. However, in some more complex cases, chatbots are integrated with existing third-party solutions (e.g., integrating IBM Watson in the back-end [31]). The majority of the chatbots have been implemented using Python libraries. Concerning the front-end, they use either customized implementations or rely on existing platforms such as Telegram [13] and Facebook messenger [1,2,17,32].

\section{SRQ10: Benefits}

The advantages brought by CBT are multiple (e.g., time- and quality-wise) and measurable. The perception of time is very important in the tourism sector, and it has a great influence on customer satisfaction. Chatbots are perceived as a 24/7 working concierge always available and providing instant support $[4,12]$. Chatbots reduce and simplify the human-machine interaction process (i.e., $\backsim 80 \%$ of all customer requests are automatically processed, delegating to the human personnel only the remaining $\sim 20 \%$ [33]). Chatbots can usually undertake numerous simultaneous and personalized conversations - only limited by the hosting machine [4]. The quality of the service provided is constant, and it is not affected by common employee-related risks (i.e., strikes, discrimination, quitting the job with no notice, showing negative emotions, shirk from work, and getting ill $[4,19])$. To date, despite explicit or implicit ethics implementation [3], no chatbot on the market has raised complaints about its fairness or misconduct. Conversely, in some cases, the tests have indicated that the responses generated by the bots have been perceived as more empathetic than those provided by human agents, thus raising customer appreciation [18].

Indeed, CBs have received positive feedback for the dynamic dissemination of various information, services, or narrative content (textual and multimedia), which has made possible to integrate and adapt them to the users' needs and dynamic behavior, rarely raising questions on the respect of the users' privacy $[1,11,31]$. Another highly appreciated advantage is a short time required 
to perform sophisticated analysis. Enabling a prompt understanding of the customer requirements can enable prompt predictions and more accurate replies and overall interactions [29]. Finally, chatbots provide tangible financial benefits. For example, savings employees' time from tedious and repetitive tasks [21] -therefore contributing to reduce personnel demand and staff workload $[12,27]-$, automatizing the advertising activities, recording growth in sales, and, overall, increasing the brand's value [27].

\section{SRQ11: Drawbacks}

The limitations elicited from the primary studies can be grouped into 3 categories (i.e., user-, provider- and system-related). Concerning the providers, small businesses have significant difficulties in fitting the design, development, and maintenance of CB into their business plan. Hence, as mentioned above, costs can start from $\$ 30,000$ and up to $\$ 150,000$ if more complex analysis and integration with third-party services are required [19,27,32]. A given chatbot could be the only way to contact the service provider, representing the single point of failure of the communication, raising frustration and delusion in the user.

Concerning the chatbot limitations, the incapability of processing complex information, providing scattered and artificial/unnatural interactions, looping on inappropriate suggestions, and the difficulty to interpret (in)satisfaction (e.g., sarcasm) still foster reluctance on the employment of these technologies [4]. The users might also need to share private information about their complaints or financial situation (considered sensitive), which raises the fear of having them compromised or, if possibly misinterpreted, misused [4]. Moreover, current chatbots have been criticized for the lack of creativity, involvement, and personal touch, especially in the case of misinterpretations of a request $[3,4]$. Therefore, it is a common belief that chatbots will still have to rely on human intervention/supervision [19]. Finally, in certain circumstances, chatbots can be perceived as threats: either from the "powerless" misunderstood user or the service provider employees (i.e., help-desk) who see their work positions endangered $[4,29]$.

\section{SRQ12: Open Challenges}

As for the advantages, the open challenges are mainly provider-, user-, and system-related. Within the dynamics of a chatbot, the human user plays a crucial role. Nevertheless, chatbots still struggle with lexical and semantic ambiguity [37]. Therefore, the most impelling challenges are user-related. For example, aligning the CB with the user's perspective, prevent user's uncertainty and resistance [4], determining the user's perception via NLP[9], promote clarity and wording to match or compensate the users' feelings [29], pace the conversation -choosing number and length of the words- (e.g., longer words are more calming and associated with positive emotions) [29], and avoiding annoying repetitions [37]. However, humans' change their communication style when interacting with chatbots [29]. Thus, to understand to which extent a designer should chase 
the human-like feeling rather then a more clear/structured interaction is still an open question [26].

From the provider perspective, the main open challenges are to find the right trade-off between chatbot- and human-delegated tasks (i.e., managing the loss of jobs [4]) and enabling knowledge sharing [4]. In turn, realizing an effective business plan, which must generate a considerable return of interest (ROI), is considered a chatbot-delegated task [4]. Finally, considering the nature of the primary studies (more tourism-oriented than technology-oriented), the system-wise open challenges have not been fully explored. Indeed, the challenges identified by the elaborated papers focus on data extraction and data representation [18], ensure data correctness and bias-free [1], and AI-related features [33].

\section{Discussion}

Although the concept of conversational agents dates back to the 60s, modern CBTs still mirror certain aspects of that original vision [32]. While chatbots reached a remarkable degree of automation and efficiency (e.g., ordering meals and booking flights), handling sophisticated conversations has not been mastered yet. Indeed, misunderstandings and lack of user-chatbot alignment may generate distress, frustration, and skepticism on a given chatbot or on the technology itself. For example, the Henn-na Hotel (Japan) is known for having a futuristic staff mainly composed of robots. Nevertheless, in 2015 they had to "fire" $50 \%$ of their robotic workforce. The project failed to reduce costs and employees' workload. Moreover, a number of tourists reported those bots as annoying and incapable to process even simple requests [34].

Overall, the most common (entry-level) CBTs rely on rule-based interactions, for instance, exploiting standardized menus (i.e., no need to produce and parse -via NLP-custom verbal text) [20]. Although it limits remarkably the expressiveness of the conversations, this workaround limits possible errors and misunderstandings, appearing satisfying for a broad set of scenarios.

More complex chatbots perform an in-depth analysis of both provided data and the human interlocutor's profile. Advanced AI-based NLPs are not limited to understanding what the user is saying, but also strive to understand tone, mood, etc., enabling ML-based predictions. However, to have good results with ML approaches, a large amount of data are required. This process is laborious and, to date, human intense. A common objective is indeed to reduce the human implication in data extraction and pre-processing. Notwithstanding, having a deeper understanding of a tourist and his/her interests/preferences, financial capabilities, and personality can exploit ML predictions for more tailored assistance and, more importantly, shaping future interactions. Indeed, ecommunications outperformed conventional methods. Thus, in general, hotels and the tourism industry had to evolve bridging their systems messaging platforms and social media (which, in turn, have remarkably invested in developing APIs fostering the development of chatbots on their platforms). For example, Facebook Messenger counted 66.000, 100.000, and 300.000 active chatbots in 
2016, 2017 ,and 2018 respectively [28,37]. Recently, the users of the Telegram platform skyrocketed (300 million bots in 2018). The high-quality APIs and services of this platform are attracting an increasing number of businesses [30]. The Slack platform provides an early-version of CBTs, allowing the configuration of auto-replies and personal-tasks automation (i.e., reminders). However, the bot does not support conversations [15]. Finally, Whatsapp is still relatively behind (APIs development phase) w.r.t. the other big competitors [22] The investments to facilitate CBTs made by these ICT colossi reflect the CBTs hype and, in most cases, fully justified interest.

CBTs led industries operating in the tourism sector to impose their presence in this new technological competition, preserving the distinctive traits of their brand. For example, hotels are investing in virtual concierges, providing the most innovative functionalities off the shelf. CBTs added a new dimension to already harsh competition. CBTs can both strengthen or destroy customers' satisfaction, henceforth loyalty. Creativity, originality, and efficiency play a crucial role in this new quest. Rule-based chatbots are quickly becoming outdated as AI advances. Thus, chatbots that represented an initial advantage might backfire if not evolving alongside the users' expectations. For example, KLM Royal Dutch Airlines introduced a novel chatbot supporting the tourists in packing for their trip [32], via knowing the destination, date, and trip length. In [18], the authors have foreseen that the strategical transition from rule-based systems to fully NLP-based chatbots needs a touch of empathy and social engineering [14]. Indeed, their early study anticipates the benefits of this direction in terms of user satisfaction.

Summarizing, 24/7 data availability, and menu-based interactions are only the entry-level features that a modern chatbot must provide. Solving data integration, storage, and manipulation are challenges that will continuously evolve alongside the higher abstraction goals such as (i) anticipating the user, (ii) debating with both content- and tone-aware, and (iii) delineating personality traits (possibly embracing the brand etiquette and overall style). The development of both front-end and back-end functionalities will represent a remarkable investment shift in tourism and hospitality. Finally, it must be highlighted that none of the elaborated studies has addressed ethical concerns about CBs' behaviors and/or data-management plans (DMPs). Considering the sensitive nature of the data handled, tackling such aspects is impelling.

\section{Conclusions}

Chatbot technologies require considerable investments, which are a barrier for many medium-small enterprises (SMEs). However, for those who can afford the development of chatbots, providing simple menu-based solutions no longer confers a plus to the investors. Hence, the users' expectations (led and incited by technological advancements) go way beyond what only two years ago was considered cutting-edge technology. This study systematically elaborated the most relevant recent literature in studies' abstraction, demographic details, application scenarios, recipients, requirements, services realized and desired, technology, 
advantages, limitations, and open challenges, concluding with a discussion elaborated over the aggregated understanding provided by our investigation.

\section{References}

1. Amalia A, Suprayogi M (2019) Engaging millennials on using chatbot messenger for eco-tourism. In: Third international conference on sustainable innovation 2019humanity, education and social sciences (IcoSIHESS 2019), Atlantis Press

2. Arteaga D, Arenas J, Paz F, Tupia M, Bruzza M (2019) Design of information system architecture for the recommendation of tourist sites in the city of manta, Ecuador through a chatbot. In: 2019 14th Iberian conference on information systems and technologies (CISTI), pp 1-6. IEEE

3. Bozic J, Tazl OA, Wotawa F (2019) Chatbot testing using AI planning. In: 2019 IEEE international conference on artificial intelligence Testing, pp 37-44

4. Buhalis D, Yen ECS (2020) Exploring the use of chatbots in hotels: technology providers' perspective. In: Information and communication technologies in tourism 2020, pp 231-242. Springer

5. Calvaresi D, Cesarini D, Sernani P, Marinoni M, Dragoni A, Sturm A (2016) Exploring the ambient assisted living domain: a systematic review. J Ambient Intell Humaniz Comput 8:1-19

6. Calvaresi D, et al (2018) Multi-agent systems' negotiation protocols for cyberphysical systems: results from a systematic literature review. In: Proceedings of ICAART

7. Calvaresi D, Calbimonte, JP, Dubosson F, Najja, A, Schumacher M (2019) Social network chatbots for smoking cessation: agent and multi-agent frameworks. In: 2019 IEEE/WIC/ACM International Conference on Web Intelligence (WI), pp 286-292. IEEE

8. Ceccarini C, Prandi, C (2019) Tourism for all: a mobile application to assist visually impaired users in enjoying tourist services. In: 2019 16th IEEE annual consumer communications and networking conference (CCNC), pp 1-6. IEEE

9. Chaves AP (2020) Should my chatbot be register-specific? Designing appropriate utterances for tourism. In: Extended abstracts of the 2020 chi conference on human factors in computing systems, pp 1-11

10. Chrysovelidis G (2020) Designing a chatbot for tourism

11. Clarizia F, Colace F, De Santo M, Lombardi M, Pascale F, Santaniello D (2019) A context-aware chatbot for tourist destinations. In: 2019 15th international conference on signal-image

12. cvent: Hotel chatbots: Your new best friends for creating a great customer experience. https://www.socialtables.com/blog/hospitality-technology/chat-bots/

13. Dyrkolbotn S, Pedersen T, Slavkovik M (2018) On the distinction between implicit and explicit ethical agency. In: Proceedings of the 2018 AAAI/ACM conference on AI, ethics, and society, pp 74-80

14. Hadnagy C (2010) Social engineering: the art of human HackingSocial Engineering: The art of Human Hacking. Wiley, New York

15. Haque MM (2019) Slackbot design and development

16. Hashim NL, Isse AJ (2019) Usability evaluation metrics of tourism mobile applications. J Softw Eng Appl 12(7):267-277

17. Hosseini S (2020) Using a chatbot to increase tourists' engagement. LAB University of Applied Sciences 
18. Hu T et al. (2018) Touch your heart: a tone-aware chatbot for customer care on social media. In: Proceedings of the CHI conference on human factors in computing systems, pp 1-12

19. Ivanov SH (2019) Ultimate transformation: how will automation technologies disrupt the travel, tourism and hospitality industries? Zeitschrift für Tourismuswissenschaft 11(1):25-43

20. Ivanov SH (2020) The first chatbot of a tourism/hospitality journal: editor's impressions. Eur J Tour Res 24:2401

21. Ivanov SH, Webster C (2017) Adoption of robots, artificial intelligence and service automation by travel, tourism and hospitality companies-a cost-benefit analysis. Artificial Intelligence and Service Automation by Travel, Tourism and Hospitality Companies-A Cost-Benefit Analysis

22. Jindal G, Upadhyay D, Jha A (2020) Whatsapp chatbot. Technical report, EasyChair

23. Kasinathan V, Abd Wahab MH, Idrus SZS, Mustapha A, Yuen KZ (2020) Aira chatbot for travel: case study of AirAsia. In: Journal of Physics: Conference Series, vol 1529, p 022101. IOP Publishing

24. Kitchenham B, Pearl Brereton O, Budgen D, Turner M, Bailey J, Linkman S (2009) Systematic literature reviews in software engineering - a systematic literature review. Inf Softw Technol 51(1):7-15. https://doi.org/10.1016/j.infsof.2008. 09.009

25. Kitchenham B, Brereton P, Turner M, Niazi M, Linkman S, Pretorius R, Budgen D (2010) Refining the systematic literature review process-two participant-observer case studies. Empir Softw Eng 15(6):618-653. https://doi.org/10.1007/s10664-0109134-8

26. Lombardi M, Pascale F, Santaniello D (2019) An application for cultural heritage using a chatbot. In: 2019 2nd international conference on computer applications and information security (ICCAIS), pp 1-5. IEEE

27. Lukanova G, Ilieva G (2019) Robots, artificial intelligence, and service automation in hotels. Robots, Artificial Intelligence, and Service Automation in Travel, Tourism and Hospitality, pp 157-183. Emerald Publishing Limited

28. Machine $\mathrm{T}$ Facebook messenger passes 300,000 bots. https://venturebeat. com/2018/05/01/facebook-messenger-passes-300000-bots/\#: :text=Facebook \%20today\%20announced\%20that\%20its,at\%20F8\%20two\%20years\%20ago

29. Melián-González S, Gutiérrez-Taño D, Bulchand-Gidumal J (2019) Predicting the intentions to use chatbots for travel and tourism. Current Issues in Tourism

30. Morze N, Buinytska O, Varchenko-Trotsenko L (2017) Use of bot-technologies for educational communication at the University

31. phocuswire: How cutting-edge hotels use artificial intelligence for a great guest experience. https://www.phocuswire.com/How-cutting-edge-hotels-use-artificialintelligence-for-a-great-guest-experience

32. Popesku J et al (2019) Current applications of artificial intelligence in tourism and hospitality. In: Sinteza 2019-International Scientific Conference on Information Technology and Data Related Research, pp 84-90. Singidunum University

33. Quicktext: How big hospitality brands are leveraging AI today. https://www. quicktext.im/blog/how-big-hospitality-brands-are-leveraging-ai-today/

34. Quicktext: Tech japan's henn-na hotel fires half its robot workforce. https://www. hotelmanagement.net/tech/japan-s-henn-na-hotel-fires-half-its-robot-workforce

35. Ruf B, Sammarco M, Aigrain J, Detyniecki M (2020) Pharmabroad: a companion chatbot for identifying pharmaceutical products when traveling abroad. Inf Commun Technol Tour 2020:218-228 
36. Sano AVD, Imanuel TD, Calista MI, Nindito H, Condrobimo AR (2018) The application of agnes algorithm to optimize knowledge base for tourism chatbot. In: 2018 International Conference on Information Management and Technology (ICIMTech), pp 65-68. IEEE

37. Ukpabi DC, Aslam B, Karjaluoto H (2019) Chatbot adoption in tourism services: A conceptual exploration. Robots, Artificial Intelligence, and Service Automation in Travel, Tourism and Hospitality, pp 105-121. Emerald Publishing Limited

38. Weizenbaum J (1966) Eliza-a computer program for the study of natural language communication between man and machine. Commun ACM 9(1):36-45

Open Access This chapter is licensed under the terms of the Creative Commons Attribution 4.0 International License (http://creativecommons.org/licenses/by/4.0/), which permits use, sharing, adaptation, distribution and reproduction in any medium or format, as long as you give appropriate credit to the original author(s) and the source, provide a link to the Creative Commons license and indicate if changes were made.

The images or other third party material in this chapter are included in the chapter's Creative Commons license, unless indicated otherwise in a credit line to the material. If material is not included in the chapter's Creative Commons license and your intended use is not permitted by statutory regulation or exceeds the permitted use, you will need to obtain permission directly from the copyright holder.

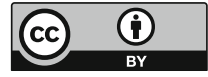

